\title{
Tissue Concentrations of Zinc, Iron, Copper, and Magnesium During the Phases of Full Thickness Wound Healing in a Rodent Model
}

\author{
Vincent Coger $^{1}$ (D) Nina Million ${ }^{2} \cdot$ Christoph Rehbock $^{2} \cdot$ Bernd Sures $^{3} \cdot$ Milen Nachev $^{3} \cdot$ Stephan Barcikowski $^{2}$. \\ Nina Wistuba ${ }^{1} \cdot$ Sarah Strauß ${ }^{1} \cdot$ Peter M. Vogt $^{1}$
}

Received: 13 June 2018 / Accepted: 30 November 2018 / Published online: 14 December 2018

(C) The Author(s) 2018

\begin{abstract}
Wound healing is a complex orchestration of processes involving cell proliferation, migration, differentiation, anabolism, and catabolism in order to restore skin continuity. Within these processes, elements such as metallic ions are involved due to their implications in cell behavior and enzymatic activity regulation. This study analyzed the kinetics of zinc, iron, copper and magnesium concentrations in a full thickness open wound rat model over 14 days. We made wounds with a diameter of $6 \mathrm{~mm}$ on the back of Lewis rats and let them heal naturally prior to analysis by histology and inductively coupled plasma mass spectrometry analysis. Histological and immunofluorescence analysis confirmed an inflammation phase until 7 days, epithelial proliferation phase from $16 \mathrm{~h}$ to 10 days, and remodeling phase from 7 days onward. These defined phases were correlated with the measured metal element kinetics. Zinc concentrations showed an inverted parabolic progression between 30.4 and a maximum of $39.9 \mu \mathrm{g} / \mathrm{g}$ dry weight. Magnesium values had a similar pattern between 283 and $499 \mu \mathrm{g} / \mathrm{g}$ dry weight. Copper concentrations, on the other hand, followed an inverted sigmoid trend with a decrease from 9.8 to $1.5 \mu \mathrm{g} / \mathrm{g}$ dry weight. Iron had a slight decrease in concentration for $24 \mathrm{~h}$ followed by an increase to a maximum of $466 \mu \mathrm{g} / \mathrm{g}$ dry weight. In conclusion, zinc, iron, and copper, even though differing in their total mass within the wound, exhibited concentration curve transitions at day 3. Interestingly, this time point correlates with the maximum proliferating keratinocyte rate during the proliferation phase.
\end{abstract}

Keywords Skin wound healing $\cdot$ Metal $\cdot$ Zinc $\cdot$ Iron $\cdot$ Copper $\cdot$ Magnesium

\section{Introduction}

Open wounds create a major impact on the healthcare system because of the financial cost of the medical treatment, pain for the patient, and emotional cost from reduced self-esteem associated with scars. Wound healing processes have been stud-

Electronic supplementary material The online version of this article (https://doi.org/10.1007/s12011-018-1600-y) contains supplementary material, which is available to authorized users.

Vincent Coger

1 Department of Plastic, Aesthetic, Hand and Reconstructive Surgery, Hannover Medical School, Hannover, Germany

2 Technical Chemistry I and Center for Nanointegration Duisburg-Essen, University of Duisburg-Essen, Duisburg, Germany

3 Aquatic Ecology and Centre for Water and Environmental Research (ZWU), University of Duisburg-Essen, Essen, Germany ied for centuries. Due to the complexity of these processes, several fields of biology and medical science are involved in understanding the physiological development, cellular processes, and enzymatic machineries that contribute to the reestablishment of skin integrity. In addition to currently studied models, a quantitative determination of the essential elements $\mathrm{Mg}, \mathrm{Cu}, \mathrm{Zn}$, and $\mathrm{Fe}$ in the wound during the skin restoration process may improve the understanding of wound healing and provide a basis for further research into this topic.

Wound healing consists of three overlapping phases, which include five processes: inflammation with hemostasis and inflammatory processes, proliferation with granulation and repidermalization processes, and finally the remodeling phase [1]. These phases are interdependent with each other and overlap in time. The duration of each wound healing phase depends on several factors [2-4]: wound type and size, age, physical condition, comorbidities, location, and wound treatment. The importance of metals has been highlighted by many studies regarding metal deficiencies and the ability to 
reestablish wound healing by metal oral supplementation [5]. Local supplementation was shown to induce several beneficial effects including acceleration of healing and strengthening of the cicatricial tissue $[6,7]$.

In the whole organism, as well as in single cells, metallostasis is precisely regulated. The dynamic relationship among the different metal elements is extremely complex and two or more of these metals can be competitors and bind to the same protein, inducing direct or indirect regulation of its activity. The binding affinities are responsible for fine regulation of enzymatic or other protein activities and ion availability. For example, copper and zinc are both susceptible to bind to metallothionein, a metal binding protein that regulates the intracellular metal availability. Zinc induces metallothionein gene transcription which was shown to reduce the half-life of the protein in comparison to copper [8].

The abilities of numerous metal ions to modify cell metabolism as well as phenotype are variable. For instance, zinc induces keratinocytes differentiation [9], magnesium supports their adhesion to laminin [10], and iron and copper induce fibroblast expression of matrix metalloproteinase-1 (MMP1) $[11,12]$. Although many studies try to use metal ions to enhance wound healing, the basic concentrations of these elements during different phases of wound healing remains a niche research field supported by scattered studies [13].

Our study focuses on four enzyme-associated elements that, based on their abundance in the literature and their success in clinical studies, are imperative for the proper course of wound healing: zinc, iron, magnesium, and copper. We describe the kinetics of these elements in the phases of natural healing of a full thickness skin wound model in rats. We characterized the cicatrization processes histologically, which allows us to correlate individual metal concentrations at different time points with the different phases of wound healing (Table 1).

\section{Materials and Methods}

\section{Animal Experiments}

All experiments were performed according to the German animal protection act and approved by the Institutional Committee on Use and Care of Animals of Hanover Medical School and the Lower Saxony State Board on Care and Use of Animals (LAVES; Oldenburg, Lower Saxony, TV 12/0993). Twenty-seven male Lewis rats (Charles River; $\mathrm{LEW} / \mathrm{Crl}$ ) with an age of 8 weeks were housed under specific pathogen-free condition with 12-h day-night cycle, with food and water ad libitum. Animals were randomly divided into nine time-groups $(n=3): 8$ and $16 \mathrm{~h}$ and $1,2,3,5,7,10$, and 14 days.
General anesthesia was induced in a narcosis chamber (Euthanex Corp.; P.O. Box 3544) ventilated at 2.5 1/min oxygen flow and 5\% Isoflurane (Baxter; HDG9623). Once asleep, masks were placed on the rats and the Isoflurane concentration was diminished to $3 \%$. The dorsal area was shaved and disinfected with Kodan Tinktur forte (Schültke; 25393-A). Novaminsulfon (Ratiopharm; 6882768) was administered subcutaneously ( $200 \mathrm{mg} / \mathrm{kg}$ of body weight) pre-operatively. Six whole skin biopsies (6-mm diameter) were taken with a biopsy punch (6-mm biopsy punch; Stiefel; 05.SF004.11/08) and preserved as control sample. Post-operative analgesia was maintained by dilution of $3.4 \mathrm{ml}$ Novalgin (Ratiopharm; 3530394 ) in $500 \mathrm{ml}$ of drinking water. Wounds were left and uncovered and air-exposed for the entire time to monitor the native healing process. The location of the wound prevented the rats from accessing the wounds and reopening them. Rats were housed individually to prevent cannibalization of the wounds by others. Wound contamination was reduced by replacing wood litter by dust-free wipes in sufficient amount for the rats to hide underneath. At the predefined time points, rats were anesthetized in the same manner as previously described and euthanized by cervical dislocation. To avoid contamination by surrounding bleeding, the whole dorsal skin was prepared prior to the wounds excision with 8-mm biopsy punches (8-mm biopsy punch; Stiefel; 05.SF004.11/08) including the scab. Three of the samples were fixed in $4 \%$ paraformaldehyde phosphate-buffered saline solution $(0.1 \mathrm{M} ; \mathrm{pH}=7.4)$ for histological analyses, and the other three were shock-frozen in liquid nitrogen for inductively coupled plasma mass spectrometry (ICP-MS) measurements.

\section{Human Skin}

The use of human skin tissue from plastic surgery patients undergoing reduction mammoplasty was approved by the ethical committee of Hannover Medical School (ref.no. 10552011). Patients (women; $n=3 ; 44$ years old \pm 17 ) gave their written consent for tissue donation. Human skin samples were submitted to the same protocol as described for the animal experiments.

\section{Histology}

After 7 days of fixation in $4 \%$ paraformaldehyde phosphatebuffered saline solution $(0.1 \mathrm{M} ; \mathrm{pH}=7.4)$, samples were washed with tap water for at least $3 \mathrm{~h}$ and dehydrated before paraffin embedding in a STP-120 (Mikrom) according to the previously described protocol [14]. The samples were impregnated with ethanol (Chemsolute; 2294.9010) at increasing concentrations from $50 \%$ to $99.9 \%(V / V)$, then with pure xylol (Roth; CN80.2), and finally with paraplast X-tra (Leica; 39603002). Samples were completely cut with a microtome (Mikrom) perpendicularly to the epidermis from side-to-side 
Table 1 Evolution of the kinetics in phases of wound healing. Results are represented as constant $(0)$, decrease (-), or increase (+) in comparison to the previous time point

\begin{tabular}{|c|c|c|c|c|c|c|c|c|c|}
\hline Tims & $1 / 3$ & $2 / 3$ & 1 & 2 & 3 & 5 & 7 & 10 & 14 \\
\hline & \multicolumn{7}{|c|}{ Inflammation } & & \\
\hline & & & \multicolumn{6}{|c|}{ Proliferation } & \\
\hline & & & & & & \multicolumn{4}{|c|}{ Remodeling } \\
\hline Wound diam & 0 & 0 & - & - & - & - & 0 & 0 & 0 \\
\hline Epidermalization & 0 & 0 & + & + & + & + & 0 & 0 & 0 \\
\hline $\begin{array}{c}\text { Epidermal } \\
\text { proliferation }\end{array}$ & + & + & + & + & + & - & - & - & - \\
\hline Zinc & + & + & + & + & + & - & - & - & - \\
\hline Iron & - & - & - & + & + & - & - & - & - \\
\hline Magnesium & + & + & + & + & + & + & - & - & - \\
\hline Copper & + & + & + & 0 & - & - & - & 0 & 0 \\
\hline
\end{tabular}

into 6- $\mu \mathrm{m}$-thick slices. The center of the wound was determined as the median of the slices regarding the wound margins observed by light microscopy.

Hematoxylin-eosin (H\&E) staining was performed for light microscopy analysis as previously described [14]. After rehydration of the thin cut with xylol, ethanol, and ultra-pure water, samples were stained $5 \mathrm{~min}$ in hematoxylin (Roth; T865.3), followed by rinsing $10 \mathrm{~min}$ in running tap water, then stained 5 min with eosin-G (Roth; X883.2), and quickly dehydrated prior to embedding under a coverslide with RotiHistoFix II (Roth; T160.1). Light microscopy was performed with a Keyence BioZero with a $\times 4$ objective and set at $\times 8$ optical magnification. The entire wounds were documented for analysis.

We determined the wound width by measuring from merge to merge, and the epidermis outgrowth over the wound in millimeters by light microscopy histomorphometry of the H\&E staining. The reepithelialization was calculated by setting epidermis growth as percentage of the wound width.

Immunofluorescence detection of $\mathrm{Ki}-67$ was performed to visualize proliferating keratinocytes, coupled with nucleus DAPI staining (Vector Laboratories; H1200). To do this, slides were deparaffinized and rehydrated as described above. After an incubation for $25 \mathrm{~min}$ at $95{ }^{\circ} \mathrm{C}$ in Antigen Unmasking Solution (Vector Laboratories; H-3300), the tissue was permeated by incubation in tris-buffer-saline (TBS) at $\mathrm{pH}=7.8$ containing 0.3\% Triton- $\times 100$ (Sigma; 9002-93-1) for 5 min. Nonspecific reactions were blocked with TBS containing $2 \%$ of bovine serum albumin (BSA) for $1 \mathrm{~h}$. The primary antibody, anti-Ki-67 (Thermo Scientific; Clone SP6, rabbit monoclonal antibody; RM-9106-S0) 1:100 diluted in TBS with $1 \% \mathrm{BSA}$, was incubated overnight at $4{ }^{\circ} \mathrm{C}$ in a wet chamber. After rinsing with TBS, a biotinylated secondary antibody (polyclonal goat anti-rabbit immunoglobulins/Biotinylated
Dako; E-0432) diluted 1:200 in TBS was applied at room temperature for $90 \mathrm{~min}$, rinsed with TBS, and incubated with Vectastain ABC Kit (Vector laboratories; PK-4000) $1 \mathrm{~h}$ at room temperature according to the manufacturer's instructions and followed by 5 -min room temperature incubation under a Cy3 Tyramide solution (Perkin Elmer; SAT704A001EA). After washing, slides were covered with Vectashield Mounting Medium with DAPI (Vector Laboratories; H1200) under cover slips.

Fluorescence microscopy was accomplished with a Zeiss AxioVert $200 \mathrm{M}$ with $\mathrm{a} \times 20$ objective. The healthy epidermis of the wound edge was documented. This was determined by a decrease in density of the extracellular matrix (ECM), as well as the presence of dermal appendages like hair follicles. Ki-67-positive keratinocytes in healthy epidermis were counted and normalized to the measured length of the same studied epidermis. The results were presented as number of positive KI-67 cells per millimeter in length of epidermis $(U)$ in function of time. Image analysis was performed with Cell-D software (Olympus) after the proper calibration of the pictures.

\section{Inductively Coupled Plasma Mass Spectrometry}

After heat dehydration ( 3 days in dry chamber at $80^{\circ} \mathrm{C}$ ), samples were weighed and digested using a microwaveaccelerated reaction system (CEM, Mars 5). Each sample was placed in a fluoropolymer vessel (XP-1500 plus) and mixed with $1.3 \mathrm{ml}$ nitric acid (65\%, Suprapure, Merck) and $2.5 \mathrm{ml}$ hydrogen peroxide (30\%, Suprapure, Merck). After digestion at $600 \mathrm{~W}$, each sample solution was poured completely into a 5-ml volumetric glass flask and brought up to volume using double distilled water. The samples were stored at room temperature until measurement. 
Inductively coupled plasma mass spectrometry (ICP-MS) was used to determine the metal concentrations [15]. These measurements were carried out using a quadrupole ICP-MS system (Perkin Elmer, Elan 6000) with an auto sampler system (Perkin Elmer, AS-90). The instrument operated at 1000W plasma power, 14 1/min plasma gas flow, $0.95 \mathrm{l} / \mathrm{min}$ nebulizer gas flow, and a sample flow rate of $1 \mathrm{ml} / \mathrm{min}$ regulated by a peristaltic pump. Between each measurement, the wash time with $1 \% \mathrm{HNO}_{3}$ (Suprapure, Merck) was set to $10 \mathrm{~s}$ to avoid contaminations. The accuracy and stability of the measurements was controlled by a multi-element standard solution (ICP multielement standard IV solution, Merck) once every 10 samples. Samples were diluted 1:10 with an internal standard solution, consisting of $1 \% \mathrm{HNO}_{3}$ containing $10 \mu \mathrm{g} / \mathrm{l}$ yttrium and $10 \mu \mathrm{g} / \mathrm{l}$ thulium. A calibration was carried out with a series of 11 dilutions of a multi-element standard solution (ICP multielement standard solution, Merck). With this calibration, the concentrations of the analytes were calculated using the corresponding regression lines with a correlation factor of $\geq 0.999$.

To validate the analytical procedure, we determined the concentrations of iron, copper, and zinc in two different reference materials, DORM-3 (National Research Council (NRC) Canada) and Oyster tissue (National Institute of Standards and Technology (NIST) Gaithersburg (USA)). Additionally, we prepared five samples for ICP-MS. Each of them consisted of $25 \mathrm{mg}$ of healthy rat skin, mixed together with different concentrations of copper and zinc varying from 0 to $10 \mathrm{ppb}$ (Copper standard solution and zinc standard solution, Merck). After preparation by digestion and dilution as mentioned before, all samples were measured by ICP-MS.

\section{Statistical Analysis}

Each group data resulted from the mean of 3 rats $(n=3)$. Each rat's values were the average of its wounds values $(n=3$ for ICP-MS, Ki-67, and H-E).

Data were statistically analyzed with IBM SPSS Statistics to calculate means, standard deviation of the mean, analysis of variance (ANOVA), and Bonferroni's post hoc correction for significance determination at a 95\% confidence level. We used a Pearson correlation analysis between the different kinetics obtained.

\section{Results}

To identify the wound healing phases of the samples, we performed H\&E staining. The wound diameters (Fig. 1) as well as the epidermis progression onto the wound (Figs. 2 and 3) were measured. Despite of the use of a 6-mm diameter biopsy punch for the wounding, the embedding and microtomic processing led to an optical appearance which was larger than the

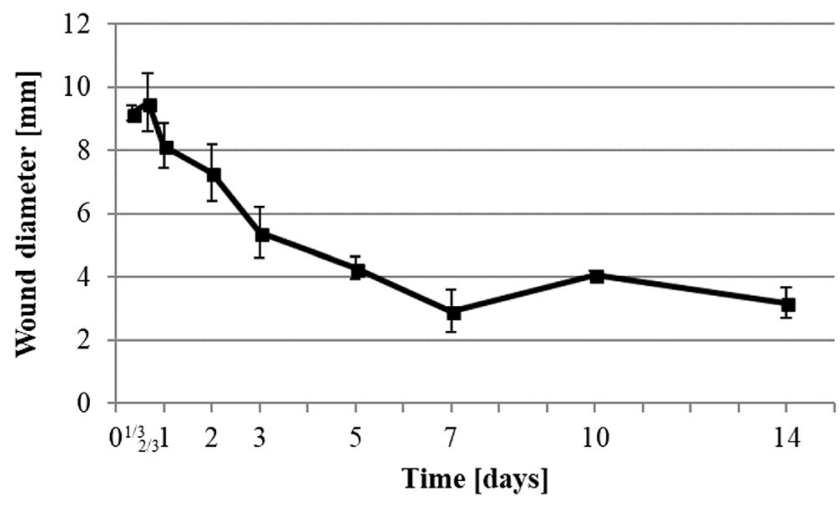

Fig. 1 Histologic measurement of wound diameter development. Data were calculated by measurements of wound margins of $H \& E$ staining of $6-\mu \mathrm{m}$ thin cut of wounds taken at the median part of the wound $\pm \operatorname{SD}(n=$ 3 rats in each group; one rats data is the mean of 3 wounds)

initially induced wound diameter (Fig. 1). The wounds remained stable in diameter for 16 -h post-operation with a diameter of $9 \mathrm{~mm} \pm 0.5 \mathrm{~mm}$. Afterwards, the diameter of the wound rapidly decreased until day $7(2.9 \mathrm{~mm} \pm 0.7)$. A slight relaxation to $4.1 \mathrm{~mm} \pm 0.1$ could be observed at day 10 before the diameter reached $3.2 \mathrm{~mm} \pm 0.5$ at day 14 .

H\&E staining visualized the reepithelialization process of the wound (Figs. 2 and 3), starting between 16 and $24 \mathrm{~h}$ after wounding. Once the reepithelialization started, the process continued at a rate of $0.33 \mathrm{~mm}$ per day until day 7 by which the epidermis covered $100 \%$ of the wound (Fig. 3). Due to the above-mentioned relaxation of the wound diameter at the 10th day (Fig. 1), we could still measure an increase in the length of the epidermis in the wound at day 10 (Fig. 2), even if it had fully covered the wound site from day 7 .

We also performed immune-fluorescence staining against $\mathrm{Ki}-67$ and counted the number of positive Ki-67 keratinocytes per millimeter of epidermis $(U)$ in the wound margin $(85 \mu \mathrm{m}$ \pm 27 ) (Fig. 4). Keratinocyte proliferation increased from the 16 th hour up to a maximum of $30 U \pm 15$ on the 3 rd day. Then

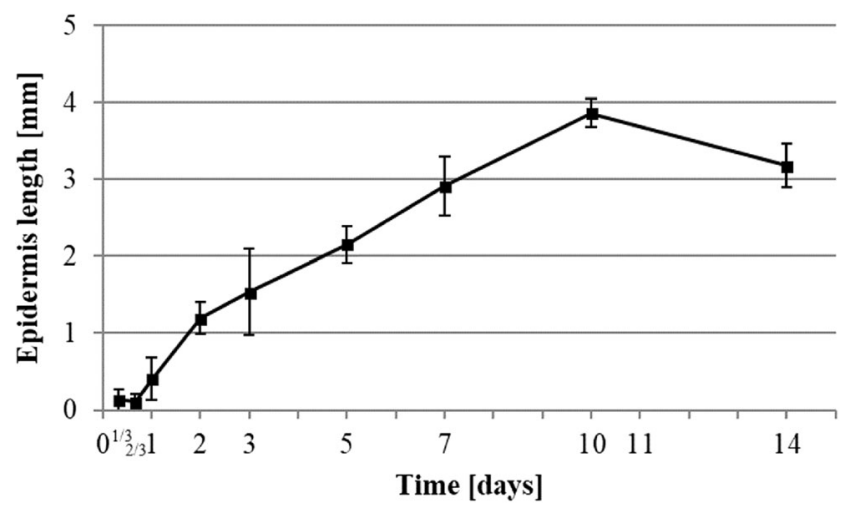

Fig. 2 Histologic measurement of epidermis development. Data were calculated by measurement of growing epidermis from the margins of H\&E staining of $6-\mu \mathrm{m}$ thin cut of wounds taken at the median part of the wound $\pm \mathrm{SD}$ ( $n=3$ rats in each group; one rats data is the mean of 3 wounds) 


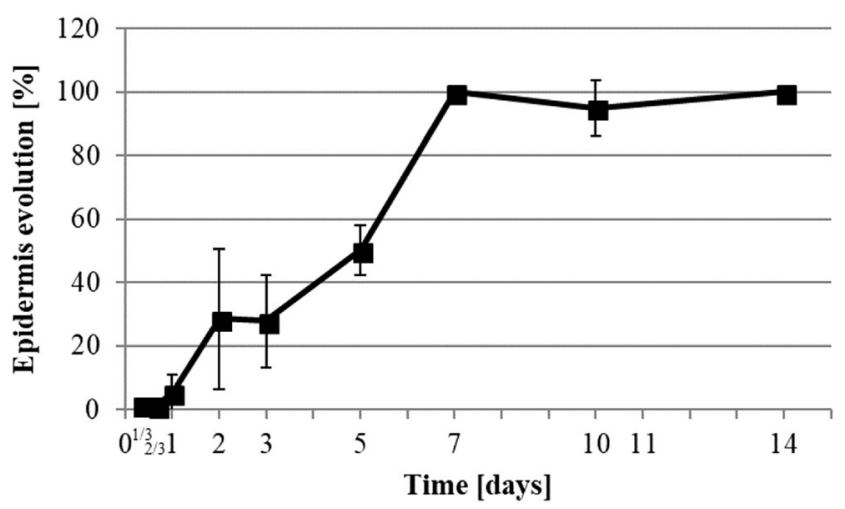

Fig. 3 Histologic measurement of reepithelialization relative to the wound diameter. Data were calculated by measurement of growing epidermis from the margins normalized to the wound diameter of H\&E staining of 6- $\mu \mathrm{m}$ thin cut of wounds taken at the median part of the wound $\pm \mathrm{SD}$ ( $n=3$ rats in each group; one rats data is the mean of 3 wounds)

the relative number of proliferative keratinocytes decreased slowly, but its level at the 14th day was still higher compared to healthy skin $(163 \pm 72$ versus $33 \pm 21 U P=0.02)$.

After acidic degradation of the dried wound samples (mean dry weight of the samples $30.7 \pm 4.0 \mathrm{mg}$ ), metal elements were quantified by ICP-MS. As a baseline, we determined the concentration of those elements in the healthy skin that we excised with biopsies (mean dry weight of the samples $14.3 \pm$ $3.1 \mathrm{mg}$ ) in order to set the wounds (Table 2). We also performed ICP-MS on human skin obtained from reduction mammoplasty (mean dry weight of the samples $33.0 \pm$ $14 \mathrm{mg}$ ). We noticed two concentration ranges: magnesium and iron which were higher than $100 \mu \mathrm{g} / \mathrm{g}$ of dry weight and zinc and copper that were below $50 \mu \mathrm{g} / \mathrm{g}$ of dry weight.

Healthy skin of rats showed a baseline level of zinc of 39.1 $\pm 7.3 \mu \mathrm{g} / \mathrm{g}$ of dry weight (Table 2 and Fig. 5). In the wounds, level of zinc was lower with $30.4 \pm 2.1 \mu \mathrm{g} / \mathrm{g}$ at $8 \mathrm{~h}$, reached the baseline level of $39.9 \pm 1.7 \mu \mathrm{g} / \mathrm{g}$ of dry weight between days 2

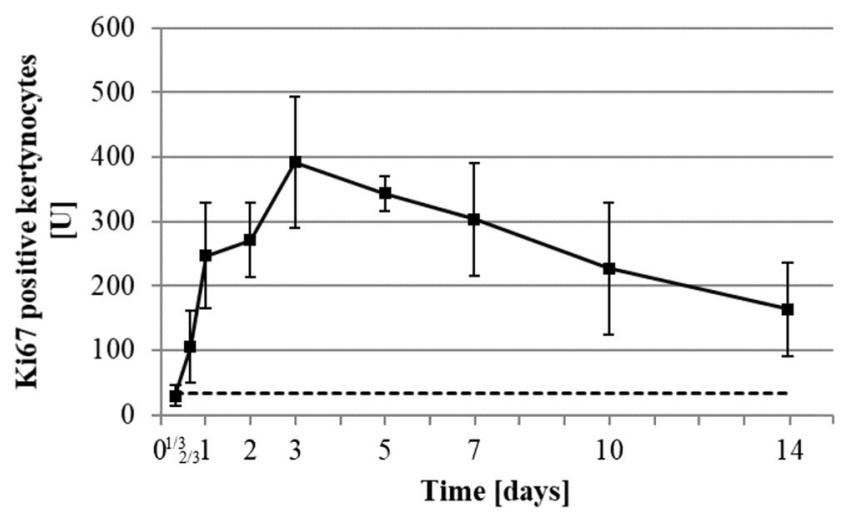

Fig. 4 Immunofluorescence measurement of number of Ki-67-positive keratinocytes at the wound margins in time in relation to the observed epidermis length. Dot line represents healthy skin baseline $(0.03 \mathrm{U} \pm$ $0.02)$. All data were displayed as mean $\pm \mathrm{SD}$ of three rats $(n=3$ rats in each group; one rat's data is the mean of 3 wounds) and 3 , and then progressively decreased to $31.2 \pm 1.6 \mu \mathrm{g} / \mathrm{g}$ of dry weight until day 14 post-operation (Fig. 5).

The iron concentration (Fig. 6) dropped down in the first $24 \mathrm{~h}$ from $164 \pm 12$ to $129 \pm 22 \mu \mathrm{g} / \mathrm{g}$ and rapidly increased to $467 \pm 131 \mu \mathrm{g} / \mathrm{g}$ on day 3 . Then it decreased slowly to reach $116 \pm 9 \mu \mathrm{g} / \mathrm{g}$ on day 14 . The magnesium concentration (Fig. 7) slowly increased from a baseline of $332 \pm 25$ to 513 $\pm 53 \mu \mathrm{g} / \mathrm{g}$ of dry weight within the first 5 days followed by a decrease to $283 \pm 16 \mu \mathrm{g} / \mathrm{g}$ on day 14 .

The temporal course of copper concentration (Fig. 8) showed an increase from $9.1 \pm 4.1$ to $13.8 \pm 3.2 \mu \mathrm{g} / \mathrm{g}$ in the first 2 days before a drastic reduction below $2 \mu \mathrm{g} / \mathrm{g}$ between days 3 and 5 $(1.6 \pm 1.1$ and $0.5 \pm 0.2 \mu \mathrm{g} / \mathrm{g}$ respectively). It then remained stable under $1.5 \mu \mathrm{g} / \mathrm{g}$ until the end of the experiment.

We searched for correlation between the kinetics by calculating the Pearson correlation coefficient (PCC). Due to the biological variance, the kinetics were considered to correlate when the PCC was less than or equal to -0.8 or greater than or equal to 0.8 . The kinetics of the different metal element concentrations, the wound diameter, the wound reepithelialization, and the number of positive-Ki-67 keratinocytes per millimeter of epidermis were analyzed for correlations. We found a statistically relevant inverted correlation between the wound diameter and the reepithelialization of $-0.88(P<0.001)$. Of the metal contents, only copper showed significant correlations with the wound diameter and the reepithelialization of 0.86 and -0.83 respectively $(P<0.001)$. As the percentage of reepithelialization results from the normalization of the length of reepithelialization relative to the wound diameter, correlation between these parameters was not calculated.

Rat skin magnesium content was measured to be $332 \pm$ $25 \mu \mathrm{g} / \mathrm{g}$ of dry weight, whereas published literature states it between 10 and $150 \mu \mathrm{g} / \mathrm{g}$ in human skin (magnesium content for human skin not measured in our study) [16]. Iron level in human and rat skin was measured respectively at $150 \pm 44$ and $76.0 \pm 41 \mu \mathrm{g} / \mathrm{g}$ of dry weight, but were not significantly different $(P=0.46)$. Copper was measured at $2.8 \pm 0.1$ and $9.1 \pm$ $4.1 \mu \mathrm{g} / \mathrm{g}$ of dry weight in human and rat skin, respectively. Zinc was measured at $28.9 \pm 3.0$ and $39.1 \pm 7.3 \mu \mathrm{g} / \mathrm{g}$ of dry weight $(P=0.23)$ in human and rat skin, respectively. The difference between human and rat skin content was significant for copper concentration $(P<0.01)$ (Table 2).

\section{Discussion}

\section{Inflammation}

The inflammation phase aims to avoid physiological disturbances by first reestablishing hemostasis, counteracting any pathogens, and starting debridement of the wound bed. [1]. As skin lesions trigger wound healing, we assume the 
Table 2 Concentration baselines in rat and human healthy skin. ICP-MS results of the mean of at least 3 samples \pm SD. * [16]

\begin{tabular}{lllll}
\hline Baselines & Magnesium & Iron & Copper & Zinc \\
\hline Human & N/A & $149.9 \pm 43.5 \mu \mathrm{g} / \mathrm{g}$ & $2.8 \pm 0.11 \mu \mathrm{g} / \mathrm{g}$ & $28.9 \pm 2.98 \mu \mathrm{g} / \mathrm{g}$ \\
Rat & $\begin{array}{l}\left(10 \text { to } 150 \mu \mathrm{g} / \mathrm{g}^{*}\right) \\
331.7 \pm 24.7 \mu \mathrm{g} / \mathrm{g}\end{array}$ & $76.0 \pm 40.9 \mu \mathrm{g} / \mathrm{g}$ & $9.1 \pm 4.14 \mu \mathrm{g} / \mathrm{g}$ & $39.1 \pm 7.32 \mu \mathrm{g} / \mathrm{g}$ \\
\hline
\end{tabular}

inflammation phase to start at the very beginning of the experiment. According to published literature, we estimated the inflammation phase would decline with the progression of reepithelialization [17]. The inflammation takes place at the open area of the wound between the keratinocyte migration front that reduces the exposed area from day 1 until day 7 (Fig. 2).

\section{Proliferation}

The proliferation phase is composed of two main processes: epithelialization involving keratinocyte proliferation and migration on top of the wound bed, and granulation involving fibroblasts that proliferate, migrate, and synthetize ECM constituents.

\section{Granulation}

Fibroblasts from the surrounding dermis and circulating fibroblasts are induced to migrate into the fibrin clot. Once there, they proliferate and synthetize type III collagen and fibronectin $[18,19]$. This modification enables cell migration of keratinocytes onto the granulation tissue [20]. The beginning of the granulation tissue growth also marks the beginning of the proliferation phase. Keratinocytes in our trial started to migrate $24 \mathrm{~h}$ after wounding (Fig. 3), so this time can be considered as the starting point for granulation.

\section{Reepithelialization}

The final step of wound healing restores the impermeability of the skin barrier by reepithelialization. The pattern of keratinocytes is a reliable observation that can be used to define the evolution of wound healing and its phases [1]. Therefore, we defined the beginning of the proliferation phase at the point at which they began to migrate onto the wound at 1-day post-operation (Figs. 2 and 3).

The higher number of proliferating keratinocytes per millimeters in comparison of the literature [21] may be the result of the difference in the species studied, as well as the narrower epidermis length we analyzed at the margin of the rat's wounds.

\section{Remodeling}

The remodeling phase aims to replace the granulation tissue with healthier skin. This process takes a long time in comparison with the other phases. The resulting scar tends to match physical and physiological properties of the original skin. This phase occurs when the epidermis is fully restored $[1,13,22]$. In our results, full reepithelialization occurred on the 7 th day post-operation. Therefore, we can assume the remodeling phase starts at this time.

\section{Metals in the Phases of Wound Healing}

Significant difference was observed between the copper baseline values determined in rat and human healthy skin. This can be due to the differences in several parameters, aside of the species considerations: the disparity in dermal appendages including the denser hairiness of the rats; the human skin was around two times thicker than those of rats (data not shown); the location of the human samples was thoracic; whereas rat's biopsies were taken from the dorsum; in contrary to the rats used for our analysis, which have common genetic background and diet, human samples are subject to high variation. These differences have important implications for the common practice of scaling from rodents to humans but do not invalidate the kinetics we determined as the wound healing processes follow the same schema in the mammalian reign with minor variations.

\section{Zinc}

Zinc is strongly related to enzymatic reactions and is described as cofactor of more than 300 enzymes including the MMP family and transcription factors. MMP is a major proteinase family that is responsible for enzymatic digestion of ECM constituents [23, 24]. The decrease of zinc content analyzed for the first time (Fig. 5) cannot be due to substance loss as we do not see this pattern in other metal concentrations (Figs. 6, 7, and 8). Instead, we hypothesize a reduced catabolism in the first steps of wound healing as ECM enzymatic digestion occurs in a nonspecific manner during the inflammation phase. During the first phase, temporary ECM has to be secreted and self-assembled. Later, during the proliferation phase, the replacement ECM is a result of protein synthesis and secretion. At this stage of wound healing, degradation of the ECM is more specific and replaces fibronectin with type III collagen. The rise of zinc concentration from $8 \mathrm{~h}$ to 3 days (Fig. 5) may induce the keratinocyte proliferation during the same time, as it has been shown that keratinocyte proliferation and differentiation are controlled by zinc $[25,26]$. 


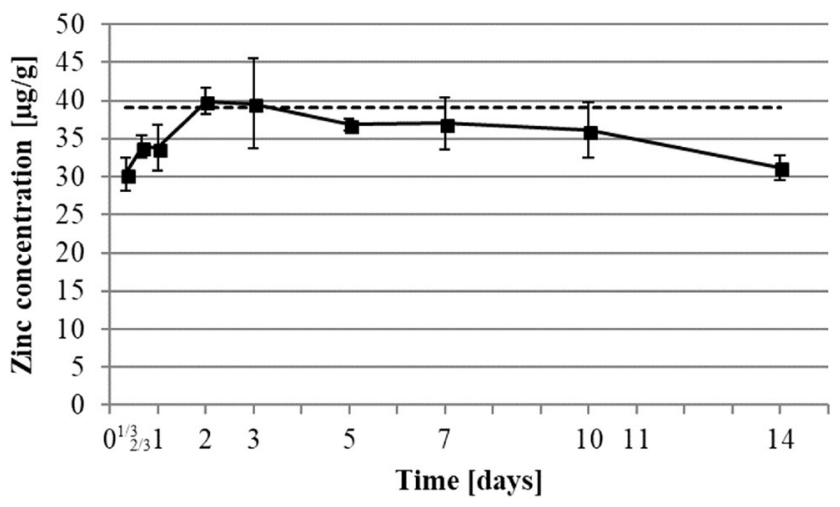

Fig. 5 Development of zinc concentration in healing wound. Zinc element was determined by ICP-MS. Dot line represents healthy skin baseline. All data were displayed as mean \pm SD of three rats $(n=3$ rats in each group; one rat's data is the mean of 3 wounds)

\section{Iron}

Iron, aside of being the active center of hemoglobin, is actively implicated in cell metabolism, cell respiration, DNA synthesis, cell cycle control, and apoptosis triggering [27]. Our results showed enhanced iron concentrations during the whole experiment in comparison with healthy skin baseline (Table 2 and Fig. 6). Only a slight initial decrease from $8 \mathrm{~h}$ to 1 day was observed (Fig. 6). It is possible that the high level at the start of the experiment resulted from the bleeding at wound sites followed by phagocytosis and debridement of erythrocytes trapped in the clot. In the early stage of healing, phagocytosis of erythrocytes by macrophages leads to an elevation of their intracellular iron concentration. This induces their activation into pro-inflammatory macrophages (M1). M1 stimulate proliferation and migration of other cell types while retarding their differentiation [28]. Iron is also a regulator of the inflammatory response induced by epithelial cells through the secretion of lactoferrin [29]. Afterwards, iron concentration increased and reached a peak at day 3 (Fig. 6), which was the time we determined to be the peak of proliferation phase. Since iron is the active center of pro-collagen lysyl-hydroxylases, it is actively involved in the maturation of pro-collagen into a stabilized collagen triple helix [30]. Therefore, the high levels of iron during the mid-proliferation phase may participate in cell metabolism for proliferation and migration, as well as the anabolism of collagen. It was also noted that during the proliferation phase, neovascularization occurs, forming new blood vessels into the wound and thus reintroducing the granulation tissue to blood and iron that constitutes hemoglobin [4]. This can be correlated with the iron concentration increase observed from day 1 to day 3 (Fig. 6).

\section{Magnesium}

Several studies implicated magnesium in various cell behaviors like proliferation, differentiation, and migration [27, 31],

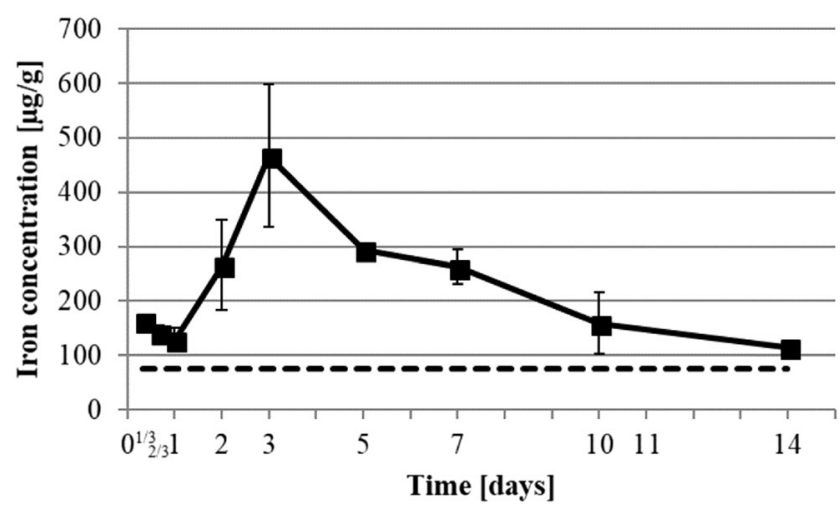

Fig. 6 Development of iron concentration in healing wound. Iron element was determined by ICP-MS. Dot line represents healthy skin baseline. All data were displayed as mean \pm SD of three rats $(n=3$ rats in each group; one rat's data is the mean of 3 wounds)

which are essential for wound healing processes. Also, extracellular levels of magnesium were proven to have direct effect on inflammation process [32]. In our model, the level of magnesium increased continuously from the skin baseline from the beginning of the experiment until the 5th day (Fig. 7). This increased concentration can be attributed to increased cell metabolism, which is needed during the proliferation phase. A peak was observed 5 days after wounding (Fig. 7). This corresponds to the peak of the proliferation phase between $24 \mathrm{~h}$ and 7 days. As previously described, the formation of celldense populations by rapid proliferation and deposition of collagen and fibronectin occurs during this phase. After this time, the proliferation phase decreases as the remodeling phase starts, and the magnesium level drops down. Energyconsuming cell behaviors like proliferation, differentiation, and migration are no longer required. The synthesis of definitive type I collagen and rearrangement of the ECM remains, which can occur without the required urgency of repair of a wounded site [1].

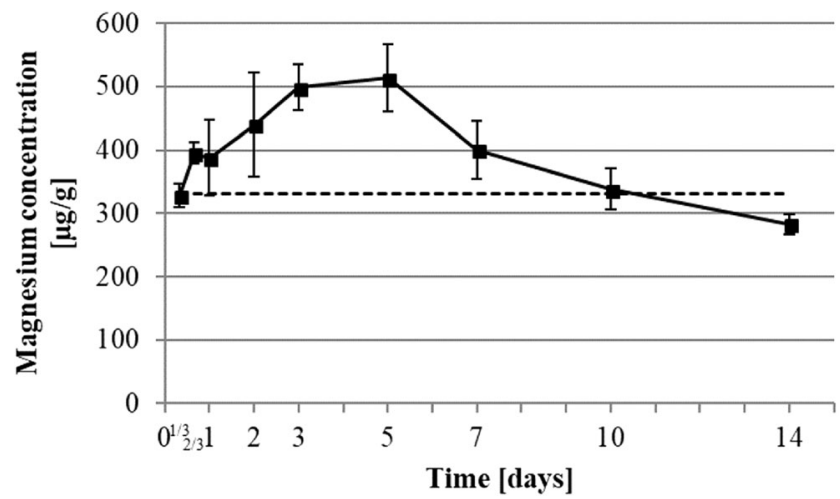

Fig. 7 Development of magnesium concentration in healing wound. Magnesium element was determined by ICP-MS. Dot line represents healthy skin baseline. All data were displayed as mean \pm SD of three rats ( $n=3$ rats in each group; one rat's data is the mean of 3 wounds) 


\section{Copper}

Several studies underlined the ability of copper to enhance, ease, or reestablish healing of wounds by enhancing cell metabolism and proliferation [6]. A key regulator of copper intracellular metallostasis, the adenosine triphosphatase 7A (ATP-7A), is induced by plateletderived growth factor (PDGF) released by the activated platelets [33]. Therefore, the observed initial augmentation of copper concentrations (Table 2 and Fig. 8) may result from an induced recruitment of copper at the wound site triggered by platelet degranulation. At the beginning of the proliferation phase, angiogenesis is strongly induced [1]. Intracellular copper concentration was shown to be a prerequisite for enabling angiogenesis induction via hypoxiainducible factor-1 (HIF-1) activation of the VEGF transcription [6]. The increase in copper concentration we observed from the beginning of the experiment to day 2 (Fig. 8) might result in the neovascularization process as the granulation tissue begins to form. The kinetics of copper were slightly different compared to previous studies conducted by Lansdown et Al. [13], in which copper concentrations increased from healthy skin baseline to rapidly reach this level within 2 days and remained stable along the 10 days of experimentation. This might result from the differences in wounding design, as Lansdown et Al. [13] performed incisional full thickness wound sutured with no tissue loss, in contrary to our wound model. As the proliferation phase occurred from day 1 to 7 , so did the granulation tissue neovascularization, and the reduction of copper concentration may be part of the slowing down of angiogenesis. It also seems to be combined with regulations that cause a rise of the zinc concentration.

Zinc and copper kinetics are interdependent due to their respective binding ability to the same proteins. This competition induces fine regulation of metal levels and, as the

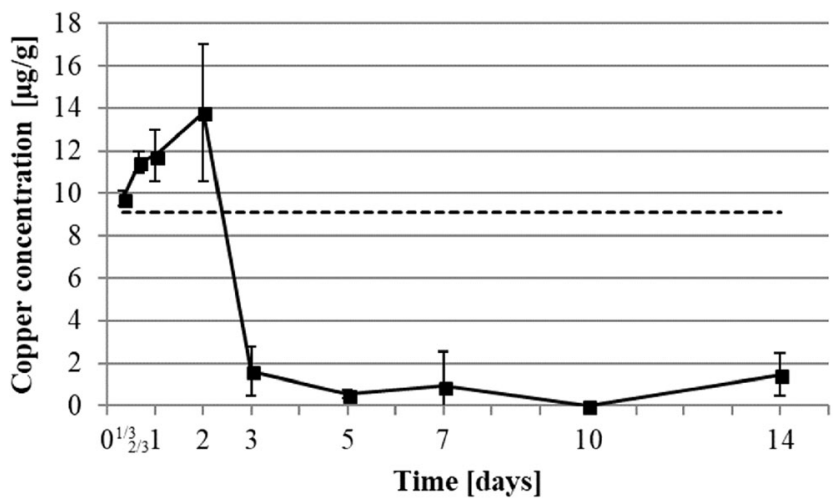

Fig. 8 Development of copper concentration in healing wound. Copper element was determined by ICP-MS. Dot line represents healthy skin baseline. All data were displayed as mean \pm SD of three rats $(n=3$ rats in each group; one rat's data is the mean of 3 wounds) presence of copper is known to reduce zinc availability, we can hypothesize that the decrease in copper content may result from the elevation of zinc concentration and contributes to higher zinc bioavailability [8]. The in situ decrease in copper concentration (Fig. 8) between days 2 and 14 post-operatively could contribute to zinc availability for cell metabolism, migration, and proliferation, as well as ECM synthesis and digestion.

On the other hand, the profile of copper kinetics differs radically. In comparison to the healthy skin base, after slightly rising, it drops down after day 2 and ends at a tenth of the healthy skin concentration on day 7 (Fig. 8). This decrease correlates with the reepithelialization process which leads the evolution of the proliferation phase [1], so copper seems to play an important part in the early stage of wound healing and inflammation [34]. This can also result from the controlled environment that reduces the infection possibility and thus, the host inflammatory response. Moreover, the copper concentration obtained at day 14 post-operation (Fig. 8) leads us to the assumption that the wounds are not fully healed. Further studies should include longer trial times that include late scar formation.

In summary, we were able to describe two metal concentration profiles. The first profile included zinc, iron, and magnesium, which reached their maximums during the proliferation phase. The second profile is copper, which shortly after the beginning of the proliferation phase decreased drastically. It is notable that, apart from magnesium, none of the metals we quantified reached the healthy skin concentration levels after 14 days. The differences between the kinetics measured by Lansdown et Al. [13] and our group may result from the wound type, and highlights the necessity of additional research in the field of wound healing to describe the specific kinetics for different types of wounds. Further research to establish long-term comparisons between fully stabilized scar and healthy skin should be considered, as there is no literature available about metal content of scar tissue. Moreover, refined wound content analysis, narrowed to specific regions, should be conducted in order to eliminate healthy skin bias during their determination.

\section{Conclusion}

We established and refined the kinetics of metal concentrations during the healing of open wounds in a rat model. This study offers new perspectives in the field of wound treatment, as healing might be enhanced by time-specific supplementation of iron, copper, zinc, and magnesium. According to our results, the supplementation of zinc, iron, and magnesium should be considered from the late inflammation until the mid-proliferation phase. Copper supplementation, on the 
other hand, may be restricted to the early stage of the wound healing. This work may be a first step into understanding the requirements of different elements during various wound healing phases, which may help to optimize compositions of metal ion-based ointments and wound coverages to enhance the wound healing process.

Acknowledgments The authors want to dedicate this article to Prof. Dr. Kerstin Reimers, who passed away during the completion of this study.

Source of Funding This study was financially supported by the Schwerpunktprogramm 1327 of the Deutsche Forschungsgemeinschaft (DFG). An official approval for using the data is present.

\section{Compliance with Ethical Standards}

Conflict of Interest The authors declare that they have no conflict of interest.

Ethical Approval The use of human tissue was approved by the ethical committee of Hannover Medical School (ref. no. 1055-2011). Patients gave their written consent for tissue donation.

All experiments were performed according to the German animal protection act and approved by the Institutional Committee on Use and Care of Animals of Hanover Medical School and the Lower Saxony State Board on Care and Use of Animals (LAVES; Oldenburg, Lower Saxony, TV 12/0993).

Open Access This article is distributed under the terms of the Creative Commons Attribution 4.0 International License (http:// creativecommons.org/licenses/by/4.0/), which permits unrestricted use, distribution, and reproduction in any medium, provided you give appropriate credit to the original author(s) and the source, provide a link to the Creative Commons license, and indicate if changes were made.

Publisher's Note Springer Nature remains neutral with regard to jurisdictional claims in published maps and institutional affiliations.?

\section{References}

1. Shaw TJ, Martin P (2009) Wound repair at a glance. J Cell Sci 122(Pt 18):3209-3213. https://doi.org/10.1242/jcs.031187

2. Martin P (1997) Wound healing-aiming for perfect skin regeneration. Science 276(5309):75-81

3. Freeman L, Pethe RS, Hollander J (2002) Update on wound management: evidence-based strategies for optimizing outcomes. Emerg Med Rep 23(26):315-328

4. Mirastschijski U, Jokuszies A, Vogt PM (2013) Skin wound healing: repair biology, wound, and scar treatment. Plastic surgery, 3rd edn. Elsevier Saunders, Philadelphia, pp 267-296

5. Razzaghi R, Pidar F, Momen-Heravi M, Bahmani F, Akbari H, Asemi Z (2018) Magnesium supplementation and the effects on wound healing and metabolic status in patients with diabetic foot ulcer: a randomized, double-blind, placebo-controlled trial. Biol Trace Elem Res 181(2):207-215. https://doi.org/10.1007/s12011-017-1056-5

6. Sen CK, Khanna S, Venojarvi M, Trikha P, Ellison EC, Hunt TK, Roy $S$ (2002) Copper-induced vascular endothelial growth factor expression and wound healing. Am J Physiol Heart Circ Physiol 282(5):H1821H1827. https://doi.org/10.1152/ajpheart.01015.2001
7. Alimohammad A, Mohammadali M, Mahmod K, Khadijeh S (2006) A study of the effect of magnesium hydroxide on the wound healing process in rats. Med J Islamic World Acad Sci 16(4):165-170

8. Irato P, Albergoni V (2005) Interaction between copper and zinc in metal accumulation in rats with particular reference to the synthesis of induced-metallothionein. Chem Biol Interact 155(3):155-164. https://doi.org/10.1016/j.cbi.2005.06.005

9. Inoue $\mathrm{Y}$, Hasegawa S, Ban S, Yamada T, Date Y, Mizutani H, Nakata S, Tanaka M, Hirashima N (2014) ZIP2 protein, a zinc transporter, is associated with keratinocyte differentiation. J Biol Chem 289(31):21451-21462. https://doi.org/10.1074/jbc.M114. 560821

10. Karecla PI, Timpl R, Watt FM (2009) Adhesion of human epidermal keratinocytes to laminin. Cell Adhes Commun 2(4):309-318. https://doi.org/10.3109/15419069409014206

11. Wenk J, Foitzik A, Achterberg V, Sabiwalsky A, Dissemond J, Meewes C, Reitz A, Brenneisen P, Wlaschek M, Meyer-Ingold W, Scharffetter-Kochanek K (2001) Selective pick-up of increased iron by deferoxamine-coupled cellulose abrogates the iron-driven induction of matrix-degrading metalloproteinase 1 and lipid peroxidation in human dermal fibroblasts in vitro: a new dressing concept. J Invest Dermatol 116(6):833-839. https://doi.org/10.1046/j. 1523-1747.2001.01345.x

12. Philips N, Hwang H, Chauhan S, Leonardi D, Gonzalez S (2010) Stimulation of cell proliferation and expression of matrixmetalloproteinase-1 and interluekin- 8 genes in dermal fibroblasts by copper. Connect Tissue Res 51(3):224-229. https://doi. org/10.3109/03008200903288431

13. Lansdown AB, Sampson B, Rowe A (1999) Sequential changes in trace metal, metallothionein and calmodulin concentrations in healing skin wounds. J Anat 195(Pt 3):375-386

14. Killat J, Reimers K, Choi CY, Jahn S, Vogt PM, Radtke C (2013) Cultivation of keratinocytes and fibroblasts in a three-dimensional bovine collagen-elastin matrix (Matriderm(R)) and application for full thickness wound coverage in vivo. Int J Mol Sci 14(7):1446014474. https://doi.org/10.3390/ijms140714460

15. Nachev M, Sures B (2016) Seasonal profile of metal accumulation in the acanthocephalan Pomphorhynchus laevis: a valuable tool to study infection dynamics and implications for metal monitoring. Parasit Vectors 9(1):300. https://doi.org/10.1186/s13071-0161576-4

16. Goldblum RW, Derby S, Lerner AB (1953) The metal content of skin, nails and hair*. J Investig Dermatol 20(1):13-18. https://doi. org/10.1038/jid.1953.3

17. Greenhalgh DG (1998) The role of apoptosis in wound healing. Int J Biochem Cell Biol 30(9):1019-1030

18. Grinnell F, Billingham RE, Burgess L (1981) Distribution of fibronectin during wound healing in vivo. J Investig Dermatol 76(3): 181-189. https://doi.org/10.1111/1523-1747.ep12525694

19. Makogonenko E, Tsurupa G, Ingham K, Medved L (2002) Interaction of fibrin(ogen) with fibronectin: further characterization and localization of the fibronectin-binding site. Biochemistry 41(25):7907-7913. https://doi.org/10.1021/bi025770x

20. Gorodetsky R, Vexler A, An J, Mou X, Marx G (1998) Haptotactic and growth stimulatory effects of fibrin(ogen) and thrombin on cultured fibroblasts. J Lab Clin Med 131(3):269-280

21. Agren MS, Karlsmark T, Hansen JB, Rygaard J (2001) Occlusion versus air exposure on full-thickness biopsy wounds. J Wound Care 10(8):301-304. https://doi.org/10.12968/jowc.2001.10.8.26109

22. Werner S, Krieg T, Smola H (2007) Keratinocyte-fibroblast interactions in wound healing. J Invest Dermatol 127(5):998-1008. https://doi.org/10.1038/sj.jid.5700786

23. Maret W (2013) Zinc biochemistry: from a single zinc enzyme to a key element of life. Adv Nutr 4(1):82-91. https://doi.org/10.3945/ an. 112.003038 
24. Rohani MG, Parks WC (2015) Matrix remodeling by MMPs during wound repair. Matrix Biol 44-46:113-121. https://doi.org/10.1016/ j.matbio.2015.03.002

25. Frank S, Kampfer H, Podda M, Kaufmann R, Pfeilschifter J (2000) Identification of copper/zinc superoxide dismutase as a nitric oxideregulated gene in human (HaCaT) keratinocytes: implications for keratinocyte proliferation. Biochem J 346(Pt 3):719-728

26. Tenaud I, Sainte-Marie I, Jumbou O, Litoux P, Dreno B (1999) In vitro modulation of keratinocyte wound healing integrins by zinc, copper and manganese. Br J Dermatol 140(1):26-34

27. Jenkins W, Perone P, Walker K, Bhagavathula N, Aslam MN, DaSilva M, Dame MK, Varani J (2011) Fibroblast response to lanthanoid metal ion stimulation: potential contribution to fibrotic tissue injury. Biol Trace Elem Res 144(1-3):621-635. https://doi. org/10.1007/s12011-011-9041-x

28. Sindrilaru A, Peters T, Wieschalka S, Baican C, Baican A, Peter H, Hainzl A, Schatz S, Qi Y, Schlecht A, Weiss JM, Wlaschek M, Sunderkotter C, Scharffetter-Kochanek K (2011) An unrestrained proinflammatory M1 macrophage population induced by iron impairs wound healing in humans and mice. J Clin Invest 121(3):985997. https://doi.org/10.1172/JCI44490

29. Engelmayer J, Blezinger P, Varadhachary A (2008) Talactoferrin stimulates wound healing with modulation of inflammation. $\mathrm{J}$
Surg Res 149(2):278-286. https://doi.org/10.1016/j.jss.2007.12. 754

30. Vranka JA, Sakai LY, Bachinger HP (2004) Prolyl 3-hydroxylase 1, enzyme characterization and identification of a novel family of enzymes. J Biol Chem 279(22):23615-23621. https://doi.org/10. 1074/jbc.M312807200

31. Banai S, Haggroth L, Epstein SE, Casscells W (1990) Influence of extracellular magnesium on capillary endothelial cell proliferation and migration. Circ Res 67(3):645-650

32. Rishi M, Ahmad A, Makheja A, Karcher D, Bloom S (1990) Effects of reduced dietary magnesium on platelet production and function in hamsters. Lab Investig 63(5):717-721

33. Ashino T, Sudhahar V, Urao N, Oshikawa J, Chen GF, Wang H, Huo Y, Finney L, Vogt S, McKinney RD, Maryon EB, Kaplan JH, Ushio-Fukai M, Fukai T (2010) Unexpected role of the copper transporter ATP7A in PDGF-induced vascular smooth muscle cell migration. Circ Res 107(6):787-799. https://doi.org/10.1161/ CIRCRESAHA.110.225334

34. Mirastschijski U, Martin A, Jorgensen LN, Sampson B, Agren MS (2013) Zinc, copper, and selenium tissue levels and their relation to subcutaneous abscess, minor surgery, and wound healing in humans. Biol Trace Elem Res 153(1-3):76-83. https://doi.org/10. 1007/s12011-013-9658-Z 